\title{
Infinitely many solutions for the discrete Schrödinger equations with a nonlocal term
}

Qilin Xie ${ }^{1}$ and Huafeng $\mathrm{XiaO}^{2^{*}}$ (D)

"Correspondence: huafeng@gzhu.edu.cn

${ }^{2}$ School of Mathematics and Information Science, Guangzhou University, Guangzhou, China Full list of author information is available at the end of the article

\section{记) Springer}

\begin{abstract}
In the present paper, we consider the following discrete Schrödinger equations

$$
-\left(a+b \sum_{k \in \mathbf{Z}}\left|\Delta u_{k-1}\right|^{2}\right) \Delta^{2} u_{k-1}+V_{k} u_{k}=f_{k}\left(u_{k}\right) \quad k \in \mathbf{Z}
$$

where $a, b$ are two positive constants and $V=\left\{V_{k}\right\}$ is a positive potential. $\Delta u_{k-1}=u_{k}-u_{k-1}$ and $\Delta^{2}=\Delta(\Delta)$ is the one-dimensional discrete Laplacian operator. Infinitely many high-energy solutions are obtained by the Symmetric Mountain Pass Theorem when the nonlinearities $\left\{f_{k}\right\}$ satisfy 4-superlinear growth conditions. Moreover, if the nonlinearities are sublinear at infinity, we obtain infinitely many small solutions by the new version of the Symmetric Mountain Pass Theorem of Kajikiya.
\end{abstract}

Keywords: Solutions; Discrete Schrödinger equations; Kirchhoff type

\section{Introduction}

In the present paper, we consider the following discrete Schrödinger equations

$$
-\left(a+b \sum_{k \in \mathbf{Z}}\left|\Delta u_{k-1}\right|^{2}\right) \Delta^{2} u_{k-1}+V_{k} u_{k}=f_{k}\left(u_{k}\right) \quad k \in \mathbf{Z},
$$

where $a, b$ are two positive constants and $V=\left\{V_{k}\right\}$ is a positive potential. $\Delta u_{k-1}=u_{k}-u_{k-1}$ and $\Delta^{2}=\Delta(\Delta)$ is the one-dimensional discrete Laplacian operator.

The discrete Schrödinger equations play a significant role in many areas, such as nonlinear optics [7], biomolecular chains [11] and Bose-Einstein condensates [16]. If $a=1$ and $b=0$, problem (1) reduces to the classical discrete Schrödinger equations, which have been extensively studied by many authors in the past several decades. In Ma and Guo [17] and Zhang and Pankov [32], the authors studied the nontrivial solution of discrete Schrödinger equations with a coercive potential by variation methods. In Lin et al. [15], the authors considered a class of discrete nonlinear nonperiodic systems and obtained the existence of the homoclinic solutions. For more related works, we refer to [4-6, 18$20,25,28]$ and their references.

The problem (1) is a discrete case for a class of nonlocal problems and we call this nonlocal term a Kirchhoff type. This class of nonlocal equations is an extension of the classi-

(c) The Author(s) 2022. This article is licensed under a Creative Commons Attribution 4.0 International License, which permits use, sharing, adaptation, distribution and reproduction in any medium or format, as long as you give appropriate credit to the original author(s) and the source, provide a link to the Creative Commons licence, and indicate if changes were made. The images or other third party material in this article are included in the article's Creative Commons licence, unless indicated otherwise in a credit line to the material. If material is not included in the article's Creative Commons licence and your intended use is not permitted by statutory regulation or exceeds the permitted use, you will need to obtain permission directly from the copyright holder. To view a copy of this licence, visit http://creativecommons.org/licenses/by/4.0/ 
cal d'Alembert's wave equations because of the effects of the changes in the length of the string during the vibrations. There is a large number of papers concerning the solutions of Kirchhof-type problems in the continuous case, such as $[9,12,26,29,31]$ and their references. As for the discrete equations with a nonlocal term, Yang and Liu in [30] investigated the existence of the nontrivial solutions via critical groups. In [3], Chakrone et al. considered the multiplicity results for a $p$-Laplacian discrete problem with Neumann boundary conditions. For more related works, we refer to [1, 2, 8, 13, 14, 22-24, 34] and their references. Recently, Wu and Huang [27] have studied the statistical solutions for nonautonomous discrete Klein-Gordon-Schrödinger-type equations.

Most of the previous works have been concerned with boundary value problems, while little has been done in discrete Schrödinger equations of the Kirchhoff type. We will consider the existence of infinitely many nontrivial solutions for this class of discrete equations. In this paper, the potential $V$ satisfies the coercive condition:

$\left(V_{1}\right) \quad V=\left\{V_{k}\right\}$ is positive and $\lim _{|k| \rightarrow+\infty} V_{k}=+\infty$.

Set $L:=-\Delta^{2}+V$, where $L u_{k}=-\Delta^{2} u_{k-1}+V_{k} u_{k}$, then $L$ is a self-adjoint operator and unbounded in $l^{2}$. With the help of the condition $\left(V_{1}\right)$, the authors in [32] proved that the spectrum $\sigma(L)$ is discrete going to $+\infty$ and we assume that $\lambda_{i}$ are the eigenvalues of $L$. If we set that

$$
E=\left\{u \in l^{2}: \sum_{k \in \mathbf{Z}} V_{k}\left|u_{k}\right|^{2}<+\infty\right\}
$$

then $E$ is a Hilbert space, in which the norm and the inner product are defined by

$$
\|u\|:=\left\{\sum_{k \in \mathbf{Z}}\left(\left|\Delta u_{k-1}\right|^{2}+V_{k}\left|u_{k}\right|^{2}\right)\right\}^{1 / 2} \quad \text { and } \quad\langle u, v\rangle_{E}=\sum_{k \in \mathbf{Z}}\left(\Delta u_{k-1} \Delta v_{k-1}+V_{k} u_{k} v_{k}\right) \text {, }
$$

respectively. Denote the standard norms of $l^{q}$ by $\|\cdot\|_{q}$ for $q \in[2,+\infty]$. First, we consider the 4-superlinear nonlinearities as follows:

$\left(f_{1}\right) f_{k} \in C(\mathbf{R}, \mathbf{R})$ and there exist $C>0$ and $p>2$ such that

$$
\left|f_{k}(t)\right| \leq C\left(|t|+|t|^{p-1}\right) \quad \text { for any } k \in \mathbf{Z} \text { and } t \in \mathbf{R} \text {. }
$$

$\left(f_{2}\right) \lim _{|t| \rightarrow+\infty} \frac{F_{k}(t)}{t^{4}}=+\infty$ uniformly in $k$, where $F_{k}(t):=\int_{0}^{t} f_{k}(s) d s$.

$\left(f_{3}\right)$ There exists $\alpha>0$ such that

$$
F_{k}(t) \leq \frac{1}{4} f_{k}(t) t+\alpha t^{2} \quad \text { for any } k \in \mathbf{Z} \text { and } t \in \mathbf{R} \text {. }
$$

$\left(f_{3}^{\prime}\right)$ There exists $L>0$ such that

$$
F_{k}(t) \leq \frac{1}{4} f_{k}(t) t \quad \text { for any } k \in \mathbf{Z} \text { and }|t| \geq L
$$

$\left(f_{4}\right) f_{k}(-t)=-f_{k}(t)$ for any $k \in \mathbf{Z}$ and $t \in \mathbf{R}$.

If $u=\left\{u_{k}\right\}$ satisfies problem (1) for any $k \in \mathbf{Z}$, we call that $u$ a solution of this problem. Moreover, if $u \neq 0$, we call that $u$ a nontrivial solution. With the above assumptions $\left(V_{1}\right)$ 
and $\left(f_{1}\right)$, the energy functional $I: E \rightarrow \mathbf{R}$ is well defined by

$$
I(u)=\frac{1}{2} \sum_{k \in \mathbf{Z}}\left(a\left|\Delta u_{k-1}\right|^{2}+V_{k} u_{k}^{2}\right)+\frac{1}{4} b\left(\sum_{k \in \mathbf{Z}}\left|\Delta u_{k-1}\right|^{2}\right)^{2}-\sum_{k \in \mathbf{Z}} F_{k}\left(u_{k}\right) .
$$

Moreover, $I$ is a class of $C^{1}(E, \mathbf{R})$ and

$$
\left\langle I^{\prime}(u), v\right\rangle=\left(a+b\|\Delta u\|_{2}^{2}\right) \sum_{k \in \mathbf{Z}} \Delta u_{k-1} \Delta v_{k-1}+\sum_{k \in \mathbf{Z}} V_{k} u_{k} v_{k}-\sum_{k \in \mathbf{Z}} f_{k}\left(u_{k}\right) v_{k},
$$

for any $u, v$ in $E$. Thus, $u$ is a critical point of $I$ in $E$ if and only if $u$ is a solution of problem (1). Now, the first result is stated concerning the infinitely many solutions when the nonlinearities are 4-superlinear growth.

Theorem 1.1 If $\left(V_{1}\right)$ and $\left(f_{1}\right)-\left(f_{4}\right)$ hold, problem (1) possesses infinitely many nontrivial solutions $\left\{u^{n}\right\}$ in $E$ with high energies, i.e., $I\left(u^{n}\right) \rightarrow+\infty$ as $n \rightarrow \infty$.

It is evident to check that $\left(f_{1}\right)$ and $\left(f_{3}^{\prime}\right)$ imply $\left(f_{3}\right)$. Hence, we have the following result.

Theorem 1.2 If $\left(V_{1}\right),\left(f_{1}\right),\left(f_{2}\right),\left(f_{3}^{\prime}\right)$ and $\left(f_{4}\right)$ hold, problem (1) possesses infinitely many nontrivial solutions $\left\{u^{n}\right\}$ in $E$ with high energies, i.e., $I\left(u^{n}\right) \rightarrow+\infty$ as $n \rightarrow \infty$.

The above two results obtained infinitely many high-energy solutions are strictly dependent on the 4-superlinear growth assumption. The standard Symmetric Mountain Pass Theorem may be invalid without the superlinear assumption. In this case, we try to establish the existence of infinitely many small solutions by the new version of the Symmetric Mountain Pass Theorem of Kajikiya in [10] when the nonlinearities $f_{k}(t)$ are sublinear at infinity. More precisely, we assume that

$\left(f_{5}\right) f_{k}(t)=\kappa \xi_{k}|t|^{\kappa-2} t$, where $\kappa \in(1,2)$ is a constant and $\xi=\left\{\xi_{k}\right\} \in l \frac{2}{2-\kappa}$.

Theorem 1.3 If $\left(V_{1}\right)$ and $\left(f_{5}\right)$ hold, problem (1) possesses infinitely many nontrivial solutions $\left\{u^{n}\right\}$ in $E$ with small energy, i.e., $I\left(u^{n}\right) \rightarrow 0^{-}$as $n \rightarrow \infty$.

In fact, we will prove a more general result than the above theorem.

Theorem 1.4 If $\left(V_{1}\right),\left(f_{4}\right)$ and the following assumption hold,

$\left(f_{6}\right)$ there exist $\kappa, \delta, \sigma \in(1,2), p>2$ and $C$ such that

$$
\xi_{k}|t|^{\kappa} \leq f_{k}(t) t
$$

and

$$
\left|f_{k}(t)\right| \leq w_{k}|t|^{\delta-1}+h_{k}|t|^{\sigma-1}+C|t|^{p-1} \quad \text { for any } k \in \mathbf{Z} \text { and } t \in \mathbf{R},
$$

where $\xi=\left\{\xi_{k}\right\}, w=\left\{w_{k}\right\}, h=\left\{h_{k}\right\}$ are three positive sequences satisfying $\xi \in l \frac{2}{2-\kappa}, w \in$ $l \frac{2}{2-\delta}$ and $h \in l \frac{2}{2-\sigma}$.

Then, problem (1) possesses infinitely many nontrivial solutions $\left\{u^{n}\right\}$ in $E$ with small energies, i.e., $I\left(u^{n}\right) \rightarrow 0^{-}$as $n \rightarrow \infty$. 
Remark 1.1 It is obvious to check that Theorem 1.4 generalizes Theorem 1.3. For example, let

$$
f_{k}(t)=\kappa \xi_{k}|t|^{\kappa-2} t+\sigma h_{k}|t|^{\sigma-2} t+C|t|^{p-2} t \quad \text { for } k \in \mathbf{Z} \text { and } t \in \mathbf{R}
$$

where $\kappa, \sigma \in(1,2), \xi=\left\{\xi_{k}\right\}$ and $h=\left\{h_{k}\right\}$ are two positive sequences satisfying $\xi \in l \frac{2}{2-\kappa}$ and $h \in l \frac{2}{2-\sigma}$. It is easy to see that $f_{k}$ satisfy all the assumptions of Theorem 1.4, but $f_{k}$ do not satisfy $\left(f_{5}\right)$.

\section{The 4-superlinear case}

The following Symmetric Mountain Pass Theorem is crucial in proving the existence of infinitely many solutions with 4-superlinear nonlinearities.

Proposition 2.1 (Symmetric Mountain Pass Theorem [21]) Let $X$ be an infinitedimensional Banach space, $X=Y \oplus Z$, where $Y$ is a finite-dimensional space. If $J \in C^{1}(X, \mathbf{R})$ satisfies the $(\mathrm{Ce})$ condition, and

$\left(J_{1}\right) J(0)=0, J(-u)=J(u)$ for all $u \in X$;

$\left(J_{2}\right)$ there exist two constants $\delta, \alpha>0$ such that $\left.J\right|_{\partial B_{\delta} \cap Z} \geq \alpha$;

$\left(J_{3}\right)$ for any finite-dimensional subspace $\widetilde{X} \subset X$, there exists $R=R(\widetilde{X})>0$ such that $J(u) \leq 0$ on $\tilde{X} \backslash B_{R}$,

then $J$ possesses an unbounded sequence of critical values.

In [21], the Symmetric Mountain Pass Theorem is established under the (PS) condition. Since the Deformation Theorem is still valid under the $(\mathrm{Ce})$ condition, we see that the Symmetric Mountain Pass Theorem also holds under the $(\mathrm{Ce})$ condition. The following embedding lemma, which follows from [17] or [33], plays a significant role in recovering the compactness result.

Lemma 2.1 If $V$ satisfies the condition $\left(V_{1}\right)$, then the embedding map from $E$ into $l^{q}$ is compact for $2 \leq q \leq+\infty$.

With the help of Lemma 2.1, we have the following compactness result.

Lemma 2.2 Under the assumption of Theorem 1.1, the functional I satisfies the (Ce) condition.

Proof Suppose that $\left\{u^{n}\right\}$ is a $(\mathrm{Ce})$ sequence,

$$
\left|I\left(u^{n}\right)\right| \leq M, \quad\left(1+\left\|u^{n}\right\|\right) I^{\prime}\left(u^{n}\right) \rightarrow 0 \quad \text { as } n \rightarrow \infty .
$$

It suffices to prove that $\left\{u^{n}\right\}$ has a converging subsequence in $E$. We first obtain that $\left\{u_{n}\right\}$ is bounded in $E$. Otherwise, $\left\|u^{n}\right\| \rightarrow+\infty$ as $n \rightarrow \infty$. Let $v^{n}=u^{n} /\left\|u^{n}\right\|$. Moving, if necessary, to a subsequence, we assume $v^{n} \rightarrow v$ in $E$, by Lemma 2.1, $v^{n} \rightarrow v$ in $l^{q}$ and $v_{k}^{n} \rightarrow v_{k}$ for any 
$k \in \mathbf{Z}$ as $n \rightarrow \infty$. There are only two cases $v=0$ or $v \neq 0$. If $v=0$, by $\left(f_{3}\right)$, we have

$$
\begin{aligned}
\frac{1}{\left\|u^{n}\right\|^{2}}(M+1) & \geq \frac{1}{\left\|u^{n}\right\|^{2}}\left(I\left(u^{n}\right)-\frac{1}{4}\left\langle I^{\prime}\left(u^{n}\right), u^{n}\right\rangle\right) \\
& \geq \frac{1}{4} \min \{a, 1\}+\frac{1}{\left\|u^{n}\right\|^{2}} \sum_{k \in \mathbf{Z}}\left(\frac{1}{4} f_{k}\left(u_{k}^{n}\right) u_{k}^{n}-F_{k}\left(u_{k}^{n}\right)\right) \\
& \geq \frac{1}{4} \min \{a, 1\}-\alpha \sum_{k \in \mathbf{Z}}\left|v_{k}^{n}\right|^{2},
\end{aligned}
$$

which implies that $\frac{1}{4} \min \{a, 1\} \leq 0$. That is impossible. If $v \neq 0$, then $\Omega_{1}:=\left\{k \in \mathbf{Z} \mid v_{k} \neq\right.$ $0\} \neq \emptyset$. For any $k \in \Omega_{1}$, we have $\left|u_{k}^{n}\right| \rightarrow+\infty$ as $n \rightarrow \infty$. By $\left(f_{2}\right)$, one obtains that for any $k \in \Omega_{1}$,

$$
\frac{F_{k}\left(u_{k}^{n}\right)}{\left|u_{k}^{n}\right|^{4}}\left|v_{k}^{n}\right|^{4} \rightarrow+\infty \quad \text { as } n \rightarrow \infty
$$

combined with Fatou's Lemma, which implies that

$$
\sum_{k \in \Omega_{1}} \frac{F_{k}\left(u_{k}^{n}\right)}{\left|u_{k}^{n}\right|^{4}}\left|v_{k}^{n}\right|^{4} \rightarrow+\infty \quad \text { as } n \rightarrow \infty
$$

It follows from $\left(f_{2}\right)$ that there exists $L_{1}>0$ such that

$$
F_{k}(t) \geq 0 \quad \text { for any } k \in \mathbf{Z} \text { and }|t| \geq L_{1} \text {. }
$$

By $\left(f_{1}\right)$, we obtain $\left|F_{k}(t)\right| \leq C t^{2} \quad$ for any $k \in \mathbf{Z}$ and $|t| \leq L_{1}$. Combining with (3), we have

$$
F_{k}(t) \geq-C t^{2} \quad \text { for any } k \in \mathbf{Z} \text { and } t \in \mathbf{R} \text {. }
$$

Hence, we obtain

$$
\sum_{k \in \mathbf{Z} \backslash \Omega_{1}} \frac{F_{k}\left(u_{k}^{n}\right)}{\left\|u^{n}\right\|^{4}} \geq-\frac{C}{\left\|u^{n}\right\|^{4}} \sum_{k \in \mathbf{Z} \backslash \Omega_{1}}\left|u_{k}^{n}\right|^{2} \geq-C \frac{\left\|u^{n}\right\|_{2}^{2}}{\left\|u^{n}\right\|^{4}} \geq-\frac{C}{\left\|u^{n}\right\|^{2}},
$$

which implies that

$$
\liminf _{n \rightarrow \infty} \sum_{k \in \mathbf{Z} \backslash \Omega_{1}} \frac{F_{k}\left(u_{k}^{n}\right)}{\left\|u^{n}\right\|^{4}} \geq 0
$$

Note that

$$
I\left(u^{n}\right)+\sum_{k \in \mathbf{Z}} F_{k}\left(u_{k}^{n}\right) \leq \frac{1}{2} \max \{a, 1\}\left\|u^{n}\right\|^{2}+\frac{b}{4}\left\|u^{n}\right\|^{4} .
$$


Dividing by $\left\|u^{n}\right\|^{4}$ on both sides and letting $n \rightarrow \infty$, we obtain

$$
\begin{aligned}
\frac{b}{4} & \geq \limsup _{n \rightarrow \infty} \sum_{k \in \mathbf{Z}} \frac{F_{k}\left(u_{k}^{n}\right)}{\left\|u^{n}\right\|^{4}} \\
& \geq \limsup _{n \rightarrow \infty}\left(\sum_{k \in \mathbf{Z} \backslash \Omega_{1}} \frac{F_{k}\left(u_{k}^{n}\right)}{\left\|u^{n}\right\|^{4}}+\sum_{k \in \Omega_{1}} \frac{F_{k}\left(u_{k}^{n}\right)}{\left\|u^{n}\right\|^{4}}\right) \rightarrow+\infty,
\end{aligned}
$$

via (2) and (4), which is impossible. In any case, we obtain a contradiction and hence $\left\{u^{n}\right\}$ is bounded in $E$.

Moving if necessary to a subsequence, we can assume $u^{n} \rightarrow u$ in $E$. It follows that

$$
\begin{aligned}
& \left\langle I^{\prime}\left(u^{n}\right)-I^{\prime}(u), u^{n}-u\right\rangle \\
& =\left(a+b\left\|\Delta u^{n}\right\|_{2}^{2}\right) \sum_{k \in \mathbf{Z}} \Delta u_{k-1}^{n} \Delta\left(u_{k-1}^{n}-u_{k-1}\right)+\sum_{k \in \mathbf{Z}} V_{k} u_{k}^{n}\left(u_{k}^{n}-u_{k}\right) \\
& \quad-\left(a+b\|\Delta u\|_{2}^{2}\right) \sum_{k \in \mathbf{Z}} \Delta u_{k-1} \Delta\left(u_{k-1}^{n}-u_{k-1}\right)-\sum_{k \in \mathbf{Z}} V_{k} u_{k}\left(u_{k}^{n}-u_{k}\right) \\
& \quad-\sum_{k \in \mathbf{Z}}\left(f_{k}\left(u_{k}^{n}\right)-f_{k}\left(u_{k}\right)\right)\left(u_{k}^{n}-u_{k}\right) \\
& =\left(a+b\left\|\Delta u^{n}\right\|_{2}^{2}\right) \sum_{k \in \mathbf{Z}}\left|\Delta\left(u_{k-1}^{n}-u_{k-1}\right)\right|^{2}+\sum_{k \in \mathbf{Z}} V_{k}\left|u_{k}^{n}-u_{k}\right|^{2} \\
& \quad-b\left(\|\Delta u\|_{2}^{2}-\left\|\Delta u^{n}\right\|_{2}^{2}\right) \sum_{k \in \mathbf{Z}} \Delta u_{k-1} \Delta\left(u_{k-1}^{n}-u_{k-1}\right) \\
& \quad-\sum_{k \in \mathbf{Z}}\left(f_{k}\left(u_{k}^{n}\right)-f_{k}\left(u_{k}\right)\right)\left(u_{k}^{n}-u_{k}\right) \\
& \geq \\
& \min \{a, 1\}\left\|u^{n}-u\right\|^{2}-b\left(\|\Delta u\|_{2}^{2}-\left\|\Delta u^{n}\right\|_{2}^{2}\right) \sum_{k \in \mathbf{Z}} \Delta u_{k-1} \Delta\left(u_{k-1}^{n}-u_{k-1}\right) \\
& \quad-\sum_{k \in \mathbf{Z}}\left(f_{k}\left(u_{k}^{n}\right)-f_{k}\left(u_{k}\right)\right)\left(u_{k}^{n}-u_{k}\right) .
\end{aligned}
$$

One has

$$
\begin{aligned}
\min \{a, 1\}\left\|u^{n}-u\right\|^{2} \leq & \left\langle I^{\prime}\left(u^{n}\right)-I^{\prime}(u), u^{n}-u\right\rangle \\
& +\sum_{k \in \mathbf{Z}}\left(f_{k}\left(u_{k}^{n}\right)-f_{k}\left(u_{k}\right)\right)\left(u_{k}^{n}-u_{k}\right) \\
& +b\left(\|\Delta u\|_{2}^{2}-\left\|\Delta u^{n}\right\|_{2}^{2}\right) \sum_{k \in \mathbf{Z}} \Delta u_{k-1} \Delta\left(u_{k-1}^{n}-u_{k-1}\right) .
\end{aligned}
$$

By the boundedness of $\left\{u^{n}\right\}$ and $u^{n} \rightarrow u$ in $E$, it is obvious that

$$
\left\langle I_{n}^{\prime}\left(u^{n}\right)-I^{\prime}(u), u^{n}-u\right\rangle \rightarrow 0 \quad \text { as } n \rightarrow \infty .
$$

By $\left(f_{1}\right)$, Lemma 2.1 and Lebesgue's dominated convergence theorem

$$
\sum_{k \in \mathbf{Z}}\left(f_{k}\left(u_{k}^{n}\right)-f_{k}\left(u_{k}\right)\right)\left(u_{k}^{n}-u_{k}\right) \rightarrow 0 \quad \text { as } n \rightarrow \infty
$$


Let us consider the functional $P: E \rightarrow \mathbf{R}$,

$$
P(w)=\sum_{k \in \mathbf{Z}} \Delta u_{k-1} \Delta w_{k-1} .
$$

Since $|P(w)| \leq\|u\|\|w\|$, we can deduce that $P$ is a continuous linear functional on $E$. By $u^{n} \rightarrow u$ in $E$, we obtain

$$
P\left(u^{n}-u\right)=\sum_{k \in \mathbf{Z}} \Delta u_{k-1} \Delta\left(u_{k-1}^{n}-u_{k-1}\right) \rightarrow 0 \quad \text { as } n \rightarrow \infty .
$$

By the boundedness of $\left\{u^{n}\right\}$ in $E$, we have

$$
b\left(\|\Delta u\|_{2}^{2}-\left\|\Delta u^{n}\right\|_{2}^{2}\right) \sum_{k \in \mathbf{Z}} \Delta u_{k-1} \Delta\left(u_{k-1}^{n}-u_{k-1}\right) \rightarrow 0 \quad \text { as } n \rightarrow \infty .
$$

It follows from (5)-(8) that $\left\|u^{n}-u\right\| \rightarrow 0$ as $n \rightarrow \infty$. Thus, $u^{n} \rightarrow u$ strongly in $E$ as $n \rightarrow \infty$.

Let $\left\{e^{j}\right\}$ be an orthonormal basis of $E$ and define $X_{j}=\operatorname{span}\left\{e_{j}\right\}, Y_{m}=\bigoplus_{j=1}^{m} X_{j}$ and $Z_{m}=$ $\overline{\bigoplus_{j=m+1}^{\infty} X_{j}}$ for any $m \in \mathbf{N}$.

Lemma 2.3 Under the assumption $\left(V_{1}\right)$, for any $2 \leq q \leq+\infty$,

$$
\beta_{m}(q):=\sup _{u \in Z_{m},\|u\|=1}\|u\|_{q} \rightarrow 0 \quad \text { as } m \rightarrow \infty
$$

Proof It is obvious that $0<\beta_{m+1}(q) \leq \beta_{m}(q)$, so that $\beta_{m}(q) \rightarrow \beta(q) \geq 0$ as $m \rightarrow \infty$. For every $m \in \mathbf{N}$, there exists $u^{m} \in \mathbf{Z}_{m}$ with $\left\|u^{m}\right\|=1$ such that

$$
\left\|u^{m}\right\|_{q}>\frac{\beta_{m}(q)}{2} .
$$

For any $w \in E, w=\sum_{j=1}^{\infty} c_{j} e_{j}$, by the Cauchy-Schwarz inequality, we have

$$
\begin{aligned}
\|\left\langle u^{m}, w\right\rangle \mid & =\left|\left\langle u^{m}, \sum_{j=1}^{\infty} c_{j} e_{j}\right\rangle\right|=\left|\left\langle u^{m}, \sum_{j=m}^{\infty} c_{j} e_{j}\right\rangle\right| \\
& \leq\left\|u^{m}\right\|\left\|\sum_{j=m}^{\infty} c_{j} e_{j}\right\|=\left\|\sum_{j=m}^{\infty} c_{j} e_{j}\right\| \rightarrow 0
\end{aligned}
$$

as $m \rightarrow \infty$, which implies that $u^{m} \rightarrow 0$ in $E$. The compact embedding of $E \hookrightarrow l^{q}, q \in$ $[2, \infty]$, implies that $u^{m} \rightarrow 0$ in $l^{q}$. Let $m \rightarrow \infty$ in (10) and we obtain $\beta_{m}(q) \rightarrow 0$ as $m \rightarrow \infty$.

The proof of Theorem 1.1 We will make use of the Symmetric Mountain Pass Theorem and Proposition 2.1 to prove Theorem 1.1. It is easy to see that $\left(J_{1}\right)$ follows from the condition $\left(f_{4}\right)$. It follows from $\left(f_{1}\right)$ that

$$
F_{k}(t) \leq c_{1} t^{2}+c_{2}|t|^{p} \quad \text { for any } k \in \mathbf{Z} \text { and } t \in \mathbf{R} \text {. }
$$


By Lemma 2.3, there exists $m \in \mathbf{N}$ large enough such that

$$
\|u\|_{2}^{2} \leq \frac{\min \{a, 1\}}{4 c_{1}}\|u\|^{2} \quad \text { and } \quad\|u\|_{p}^{p} \leq \frac{\min \{a, 1\}}{4 c_{2}}\|u\|^{p} \quad \text { for any } u \in Z_{m} .
$$

It follows from the above three inequalities that

$$
\begin{aligned}
I(u) & =\frac{1}{2} \min \{a, 1\}\|u\|^{2}-\sum_{k \in \mathbf{Z}} F_{k}\left(u_{k}^{n}\right) \\
& \geq \frac{1}{2} \min \{a, 1\}\|u\|^{2}-c_{1}\|u\|_{2}^{2}-c_{2}\|u\|_{p}^{p} \\
& \geq \frac{1}{4} \min \{a, 1\}\|u\|^{2}\left(1-\|u\|^{p-2}\right) .
\end{aligned}
$$

It follows from $p>2$ that there exist $\delta_{1}, \alpha>0$ such that $\left.I\right|_{\partial B_{\delta_{1}} \cap Z_{m}} \geq \alpha$. Thus, $\left(J_{2}\right)$ holds.

It remains to prove $\left(J_{3}\right)$. Since all norms are equivalent in a finite-dimensional space, there exists $c_{4}$ such that $\|u\|_{4}^{4} \geq c_{4}\|u\|^{4}$ for any $u \in \widetilde{E}$.

By $\left(f_{2}\right)$, for any $M>\frac{b}{4 c_{4}}$, there exists $L_{1}>0$ such that $F_{k}(t) \geq M t^{4}$ for $|t| \geq L_{1}$. It follows from $\left(f_{1}\right)$ that there exists $C_{1}>0$ such that $F_{k}(t) \geq-C_{1} t^{2}$ for $|t| \leq L_{1}$. From the above two inequalities, it follows that for any $k \in \mathbf{Z}$,

$$
F_{k}(t) \geq M t^{4}-C_{M} t^{2} \quad \text { for any } k \in \mathbf{Z} \text { and } t \in \mathbf{R} \text {, }
$$

where $C_{M}=C_{1}+M L_{1}^{2}$. It follows that

$$
\begin{aligned}
I(u) & \leq \frac{1}{2} \max \{a, 1\}\|u\|^{2}+\frac{b}{4}\|u\|^{4}-\sum_{k \in \mathbf{Z}} F_{k}\left(u_{k}\right) \\
& \leq \frac{1}{2} \max \{a, 1\}\|u\|^{2}+\frac{b}{4}\|u\|^{4}-M\|u\|_{4}^{4}+C_{M}\|u\|_{2}^{2} \\
& \leq C\|u\|^{2}-\left(M c_{4}-\frac{b}{4}\right)\|u\|^{4},
\end{aligned}
$$

for all $u \in \widetilde{E}$. Hence, there exists a large $R=R(\widetilde{E})$ such that $I(u) \leq 0$ on $\widetilde{E} \backslash B_{R}$. This completes the proof.

\section{The proof of Theorem 1.4}

To deal with the sublinear case in Theorem 1.4, we need the following Symmetric Mountain Pass Theorem of Kajikiya [10]. Let $X$ be a Banach space and $A$ be a subset of $X$. Denote by $\Gamma$ the family of closed symmetric subsets $A$ of $X$,

$$
\Gamma=\{A \in X \backslash\{0\} \mid A \text { is closed and symmetric with the origin }\} .
$$

We define the $\mathbf{Z}_{2}$-genus of $A \in \Gamma$ by $\gamma(A)$. Set $\Gamma_{m}=\{A \in \Gamma \mid \gamma(A) \geq m\}$.

Proposition 3.1 Let $X$ be an infinite-dimensional Banach space and $J \in C^{1}(X, \mathbf{R})$ satisfies the following conditions:

(a) $J(u)$ is even, bounded from below, $J(0)=0$ and $J$ satisfies the $(P S)$ condition.

(b) For any $m \in \mathbf{N}$, there exists $A_{m} \in \Gamma_{m}$ such that $\sup _{u \in A_{m}} J(u)<0$.

Then, $J$ has a sequence of critical points $\left\{u^{n}\right\}$ such that $J\left(u^{n}\right) \leq 0, u^{n} \neq 0$ and $\lim _{n \rightarrow \infty} u^{n}=0$. 
Lemma 3.1 For the finite-dimensional subspace $\widetilde{E}$ of $E$, there exists $\varepsilon>0$ such that

$$
\operatorname{meas}\left\{\left.k \in \mathbf{Z}\left|\xi_{k}\right| u_{k}\right|^{\kappa} \geq \varepsilon\|u\|^{\kappa}\right\} \geq 1 \quad \text { for any } u \in \widetilde{E} \backslash\{0\},
$$

where $\kappa$ and $\xi_{k}$ are given in $\left(f_{6}\right)$.

Proof Arguing indirectly, if not, for any positive integer $n$ there exist $u^{n} \in \widetilde{E}$ such that

$$
\operatorname{meas}\left\{\left.k \in \mathbf{Z}\left|\xi_{k}\right| u_{k}^{n}\right|^{\kappa} \geq \frac{1}{n}\left\|u^{n}\right\|^{\kappa}\right\}=0
$$

By the above inequality, we can assume that $\left\|u^{n}\right\|=1$ and

$$
\operatorname{meas}\left\{\left.k \in \mathbf{Z}\left|\xi_{k}\right| u_{k}^{n}\right|^{\kappa} \geq \frac{1}{n}\right\}=0
$$

By the compactness of the unit sphere of the finite-dimensional subspace $\widetilde{E}$, there exists a subsequence such that $u^{n} \rightarrow u^{0}$ in $\widetilde{E}$ and $u^{n} \rightarrow u^{0}$ in $l^{2}$. By the Hölder inequality, we have

$$
\sum_{k \in \mathbf{Z}} \xi_{k}\left|u_{k}^{n}-u^{0}\right|^{\kappa} \leq\left(\sum_{k \in \mathbf{Z}}\left|\xi_{k}\right|^{\frac{2}{2-\kappa}}\right)^{\frac{2-\kappa}{2}}\left(\sum_{k \in \mathbf{Z}}\left|u_{k}^{n}-u_{k}^{0}\right|^{2}\right)^{\frac{\kappa}{2}} \rightarrow 0 \quad \text { as } n \rightarrow \infty
$$

Since $\left\|u^{0}\right\|=1$, there exists $d_{1}>0$ such that meas $\left\{\left.k \in \mathbf{Z}\left|\xi_{k}\right| u_{k}^{0}\right|^{k} \geq d_{1}\right\} \geq 1$. Otherwise, one has meas $\left\{\left.k \in \mathbf{Z}\left|\xi_{k}\right| u_{k}^{0}\right|^{k} \geq \frac{1}{n}\right\}=0$, which implies that

$$
0 \leq \sum_{k \in \mathbf{Z}} \xi_{k}\left|u^{0}\right|^{\kappa+2} \leq \frac{1}{n} \sum_{k \in \mathbf{Z}}\left|u_{k}^{0}\right|^{2} \rightarrow 0 \quad \text { as } n \rightarrow \infty
$$

Thus, $u^{0}=0$, which is impossible. Set that $\Omega_{n}=\left\{\left.k \in \mathbf{Z}\left|\xi_{k}\right| u_{k}^{n}\right|^{\kappa}<\frac{1}{n}\right\}$ and $\Omega_{0}=\{k \in$ $\left.\left.\mathbf{Z}\left|\xi_{k}\right| u_{k}^{0}\right|^{\kappa} \geq d_{1}\right\}$. Thus, meas $\left(\Omega_{0} \cap \Omega_{n}\right) \geq \operatorname{meas}\left(\Omega_{0}\right)-$ meas $\left(\Omega_{n}^{c}\right) \geq 1$. Then, for $n$ large, we obtain

$$
\begin{aligned}
\sum_{k \in \mathbf{Z}} \xi_{k}\left|u_{k}^{n}-u^{0}\right|^{\kappa} & \geq \sum_{\Omega_{0} \cap \Omega_{n}} \xi_{k}\left|u_{k}^{n}-u^{0}\right|^{\kappa} \geq \frac{1}{2^{\kappa}} \sum_{\Omega_{0} \cap \Omega_{n}} \xi_{k}\left|u_{k}^{0}\right|^{\kappa}-\sum_{\Omega_{0} \cap \Omega_{n}} \xi_{k}\left|u_{k}^{n}\right|^{\kappa} \\
& \geq\left(\frac{d_{1}}{2^{\kappa}}-\frac{1}{n}\right) \operatorname{meas}\left(\Omega_{0} \cap \Omega_{n}\right) \geq\left(\frac{d_{1}}{2^{\kappa}}-\frac{1}{n}\right),
\end{aligned}
$$

which contradicts (12).

The proof of Theorem 1.4 We consider the truncated functional

$$
\varphi(u)=\frac{1}{2} \sum_{k \in \mathbf{Z}}\left(a\left|\Delta u_{k-1}\right|^{2}+V_{k} u_{k}^{2}\right)+\frac{1}{4} b\left(\sum_{k \in \mathbf{Z}}\left|\Delta u_{k-1}\right|^{2}\right)^{2}-h(\|u\|) \sum_{k \in \mathbf{Z}} F_{k}\left(u_{k}\right),
$$

for all $u \in E$, where $h \in C^{1}\left(\mathbf{R}^{+}, \mathbf{R}^{+}\right)$such that $0 \leq h \leq 1, h(t)=1$ for $0 \leq t \leq 1$ and $h(t)=0$ for $t \geq 2$. To obtain Theorem 1.4, it suffices to show that $\varphi$ possesses a sequence of critical points $\left\{u^{n}\right\}$ such that $u^{n} \rightarrow 0$ as $n \rightarrow \infty$. 
By $\left(f_{4}\right)$, we have $\varphi(-u)=\varphi(u)$. For any $u \in E$ with $\|u\|=2$, one has

$$
\varphi(u)=\frac{1}{2} \sum_{k \in \mathbf{Z}}\left(a\left|\Delta u_{k-1}\right|^{2}+V_{k} u_{k}^{2}\right)+\frac{1}{4} b\left(\sum_{k \in \mathbf{Z}}\left|\Delta u_{k-1}\right|^{2}\right)^{2} \geq \frac{1}{2} \min \{a, 1\}\|u\|^{2},
$$

which implies that $\varphi(u)$ is bounded from below and coercive. By condition $\left(f_{6}\right)$ and Lemma 2.1, we can prove that $\varphi$ satisfies the (PS) condition. For any $Y_{m}$, by Lemma 3.1, there exists $\varepsilon_{m}>0$ such that

$$
\operatorname{meas}\left\{\left.k \in \mathbf{Z}\left|\xi_{k}\right| u_{k}\right|^{\kappa} \geq \varepsilon_{m}\|u\|^{\kappa}\right\} \geq 1 \quad \text { for any } u \in Y_{m} \backslash\{0\} \text {. }
$$

Let $\Omega_{u}=\left\{\left.k \in \mathbf{Z}\left|\xi_{k}\right| u_{k}\right|^{\kappa} \geq \varepsilon_{m}\|u\|^{\kappa}\right\}$. For any $u \in Y_{m} \backslash\{0\}$, with $\|u\| \leq \min \{1$, $\left.\left(\frac{\varepsilon_{m}}{2 \kappa(a+b+1)}\right)^{1 /(2-\kappa)}\right\}$, we deduce that

$$
\begin{aligned}
\varphi(u) & \leq \frac{1}{2} \sum_{k \in \mathbf{Z}}\left(a\left|\Delta u_{k-1}\right|^{2}+V_{k} u_{k}^{2}\right)+\frac{1}{4} b\left(\sum_{k \in \mathbf{Z}}\left|\Delta u_{k-1}\right|^{2}\right)^{2}-\sum_{k \in \mathbf{Z}} F_{k}\left(u_{k}\right) \\
& \leq \frac{1}{2} \max \{a, 1\}\|u\|^{2}+\frac{b}{4}\|u\|^{4}-\frac{1}{\kappa} \sum_{k \in \mathbf{Z}} \xi_{k}\left|u_{k}\right|^{\kappa} \\
& \leq \frac{1}{2} \max \{a, 1\}\|u\|^{2}+\frac{b}{4}\|u\|^{4}-\frac{1}{\kappa} \sum_{k \in \Omega_{u}} \xi_{k}\left|u_{k}\right|^{\kappa} \\
& \leq(a+b+1)\|u\|^{2}-\frac{1}{\kappa} \varepsilon_{m}\|u\|^{\kappa} \operatorname{meas}\left(\Omega_{u}\right) \\
& \leq-(a+b+1)\|u\|^{2} .
\end{aligned}
$$

Then, choosing $0<d_{m} \leq \min \left\{1,\left(\frac{\varepsilon_{m}}{2 \kappa(a+b+1)}\right)^{1 /(2-\kappa)}\right\}$, the above inequality implies that

$$
\left\{u \in Y_{m} \mid\|u\|=d_{m}\right\} \subset\left\{u \in E \mid \varphi(u) \leq-(a+b+1) d_{m}^{2}\right\} .
$$

Setting that $A_{m}=\left\{u \in E \mid \varphi(u) \leq-(a+b+1) d_{m}^{2}\right\}$, we have

$$
\gamma\left(A_{m}\right) \geq \gamma\left(\left\{u \in Y_{m} \mid\|u\|=d_{m}\right\}\right) \geq m
$$

Hence, $A_{m} \in \Gamma_{m}$ and $\sup _{u \in A_{m}} I(u) \leq-(a+b+1) d_{m}^{2}<0$. This completes the proof.

\section{Acknowledgements}

The authors would like to thank the referees for helpful comments and valuable suggestions.

\section{Funding}

This research is supported by the National Natural Science Foundation of China (Nos. 11871171, 11701113), the Natural Science Foundation of Guangdong Province (No. 2021A1515010383), and the Project of Science and Technology of Guangzhou (No. 202102020730).

\section{Availability of data and materials}

The data and material in this paper are original.

\section{Declarations}


Authors' contributions

Both authors contributed equally to this work. All authors read and approved the final manuscript.

\section{Author details}

${ }^{1}$ School of Mathematics and Statistics, Guangdong University of Technology, Guangzhou, China. ${ }^{2}$ School of Mathematics and Information Science, Guangzhou University, Guangzhou, China.

\section{Publisher's Note}

Springer Nature remains neutral with regard to jurisdictional claims in published maps and institutional affiliations.

Received: 5 November 2021 Accepted: 20 December 2021 Published online: 14 January 2022

\section{References}

1. Aboudramane, G., Idrissa, I., Stanislas, O.: Weak homoclinic solutions to discrete nonlinear problems of Kirchhoff type with variable exponents. CUBO 19, 43-55 (2017)

2. Blaise, K., Ismaël, N., Stanislas, O.: Weak solutions to discrete nonlinear two-point boundary-value problems of Kirchhoff type. Electron. J. Differ. Equ. 2015, 105 (2015)

3. Chakrone, O., Hssini, E.L.M., Rahmani, M., Darhouche, O.: Multiplicity results for a $p$-Laplacian discrete problems of Kirchhoff type. Appl. Math. Comput. 276, 310-315 (2016)

4. Chen, G.W., Ma, S.W.: Homoclinic solutions of discrete nonlinear Schrödinger equations with asymptotically or super linear terms. Appl. Math. Comput. 232, 787-798 (2014)

5. Chen, G.W., Schechter, M.: Non-periodic discrete Schrödinger equations: ground state solutions. Z. Angew. Math. Phys. 67, 15 (2016)

6. Chen, S.T., Tang, X.H., Yu, J.S.: Sign-changing ground state solutions for discrete nonlinear Schrödinger equations. J. Differ. Equ. Appl. 25, 202-218 (2019)

7. Christodoulides, D.N., Lederer, F., Silberberg, Y.: Discretizing light behaviour in linear and nonlinear waveguide lattices. Nature 424, 817-823 (2003)

8. Erbe, L., Jia, B., Zhang, Q.: Homoclinic solutions of discrete nonlinear systems via variational method. J. Appl. Anal. Comput. 9, 271-294 (2019)

9. He, Y., Li, G.B.: Standing waves for a class of Kirchhoff type problems in $\mathbf{R}^{3}$ involving critical Sobolev exponents. Calc. Var. Partial Differ. Equ. 54, 3067-3106 (2015)

10. Kajikiya, R.: A critical point theorem related to the symmetric mountain pass lemma and its applications to elliptic equations. J. Funct. Anal. 225, 352-370 (2005)

11. Kopidakis, G., Aubry, S., Tsironis, G.P.: Targented enery transfer through discrete breathers in nonlinear systems. Phys. Rev. Lett. 87, 165501 (2001)

12. Li, G.B., Ye, H.Y.: Existence of positive ground state solutions for the nonlinear Kirchhoff type equations in $\mathbf{R}^{3}$. J. Differ. Equ. 257, 566-600 (2014)

13. Lin, G.H., Yu, J.S., Zhou, Z.: Homoclinic solutions of discrete nonlinear Schrödinger equations with partially sublinear nonlinearities. Electron. J. Differ. Equ. 96, 1 (2019)

14. Lin, G.H., Zhou, Z.: Homoclinic solutions in non-periodic discrete $\phi$-Laplacian equations with mixed nonlinearities Commun. Pure Appl. Anal. 17, 1723-1747 (2018)

15. Lin, G.H., Zhou, Z., Yu, J.S.: Ground state solutions of discrete asymptotically linear Schrödinger equations with bounded and non-periodic potentials. J. Dyn. Differ. Equ. 32, 527-555 (2020)

16. Livi, R., Franzosi, R., Oppo, G.L.: Self-localization of Bose-Einstein condensates in optical lattices via boundary dissipation. Phys. Rev. Lett. 97, 060401 (2006)

17. Ma, M.J., Guo, Z.M.: Homoclinic orbits for second order self-adjoint difference equations. J. Math. Anal. Appl. 323, 513-521 (2006)

18. Ma, M.J., Guo, Z.M.: Homoclinic orbits and subharmonics for nonlinear second order difference equations. Nonlinear Anal. 67, 1737-1745 (2007)

19. Pankov, A.: Gap solitons in periodic discrete nonlinear Schrödinger equations. Nonlinearity 19, 27-40 (2006)

20. Pankov, A., Zhang, G.: Standing wave solutions for discrete nonlinear Schrödinger equations with unbounded potentials and saturable nonlinearity. J. Math. Sci. 177, 71-82 (2011)

21. Rabinowitz, P.H.: Minimax Methods in Critical Point Theory with Applications to Differential Equations. CBMS Reg. Conf. Ser. in Math., vol. 65. Am. Math. Soc., Providence (1986)

22. Shapour, H., Ghasem, A., Johnny, A.H., Shahin, M., Giuseppe, C.: Variational approaches to p-Laplacian discrete problems of Kirchhoff-type. J. Differ. Equ. Appl. 23, 917-938 (2017)

23. Shapour, H., Ghasem, A., Shahin, M., Giuseppe, C.: Critical point approaches to difference equations of Kirchhoff-type. Differential and difference equations with applications. In: Springer Proc. Math. Stat., vol. 230, pp. 39-51. Springer, Cham (2018)

24. Shapour, H., Giuseppe, C., Amjad, S.: Perturbed Kirchhoff-type p-Laplacian discrete problems. Collect. Math. 68 , 401-418 (2017)

25. Tang, X.H.: Non-Nehari manifold method for periodic discrete superlinear Schrödinger equation. Acta Math. Sin. Engl. Ser. 32, 463-473 (2016)

26. Wang, J., Tian, L.X., Xu, J.X., Zhang, F.B.: Multiplicity and concentration of positive solutions for a Kirchhoff type problem with critical growth. J. Differ. Equ. 253, 2314-2351 (2012)

27. Wu, S., Huang, J.H.: Invariant measure and statistical solutions for non-autonomous discrete Klein-Gordon-Schrödinger-type equations. J. Appl. Anal. Comput. 10, 1516-1533 (2020)

28. Xie, Q.L.: Multiple solutions for the nonhomogeneous discrete nonlinear Schrödinger equation. Appl. Math. Lett. 91 144-150 (2019)

29. Xie, Q.L., Ma, S.W., Zhang, X.: Bound state solutions of Kirchhoff type problems with critical exponent. J. Differ. Equ 261, 890-924 (2016) 
30. Yang, J., Liu, J.: Nontrivial solutions for discrete Kirchhoff-type problems with resonance via critical groups. Adv. Differ. Equ. 2013, 308 (2013)

31. Ye, Y.W., Tang, C.L.: Multiple solutions for Kirchhoff-type equations in $\mathbf{R}^{N}$. J. Math. Phys. 54, 081508 (2013)

32. Zhang, G., Pankov, A.: Standing waves of the discrete nonlinear Schrödinger equations with growing potentials. Commun. Math. Anal. 5, 38-49 (2009)

33. Zhang, G., Pankov, A.: Standing wave solutions of the discrete non-linear Schrödinger equations with unbounded potentials. II. Appl. Anal. 89, 1541-1557 (2010)

34. Zhou, Z., Ma, D.: Multiplicity results of breathers for the discrete nonlinear Schrödinger euqations with unbounded potentials. Sci. China Ser. A 58, 781-790 (2015)

Submit your manuscript to a SpringerOpen ${ }^{\circ}$ journal and benefit from:

- Convenient online submission

- Rigorous peer review

- Open access: articles freely available online

- High visibility within the field

- Retaining the copyright to your article

Submit your next manuscript at $\gg$ springeropen.com 